This is a self-archived version of an original article. This version may differ from the original in pagination and typographic details.

Author(s): Silaev, Mikhail

Title: Finite-frequency spin susceptibility and spin pumping in superconductors with spin-orbit relaxation

Year: 2020

Version: Published version

Copyright: @ 2020 American Physical Society

Rights: In Copyright

Rights url: http://rightsstatements.org/page/lnc/1.0/?language=en

Please cite the original version:

Silaev, M. (2020). Finite-frequency spin susceptibility and spin pumping in superconductors with spin-orbit relaxation. Physical Review B, 102(14), Article 144521.

https://doi.org/10.1103/PhysRevB.102.144521 


\title{
Finite-frequency spin susceptibility and spin pumping in superconductors with spin-orbit relaxation
}

\author{
M. A. Silaev \\ Department of Physics and Nanoscience Center, University of Jyväskylä, P.O. Box 35 (YFL), FI-40014 University of Jyväskylä, Finland; \\ Moscow Institute of Physics and Technology, 141700 Dolgoprudny, Russia; \\ and Institute for Physics of Microstructures, Russian Academy of Sciences, 603950 Nizhny Novgorod, GSP-105, Russia
}

(Received 20 July 2020; revised 14 September 2020; accepted 28 September 2020; published 21 October 2020)

\begin{abstract}
Static spin susceptibility of superconductors with spin-orbit relaxation was calculated in the seminal work of Abrikosov and Gor'kov [Sov. Phys. JETP 15, 752 (1962)]. Surprisingly, the generalization of this result to finite frequencies has not been done despite being quite important for the modern topic of superconducting spintronics. The present paper fills this gap by deriving the analytical expression for spin susceptibility. The time-dependent spin response is shown to be captured by the quasiclassical Eilenberger equation with collision integrals corresponding to the ordinary and spin-orbit scattering. Using the developed formalism, we study the linear spin pumping effect between the ferromagnet and the adjacent superconducting film. The consequences for understanding recent experiments demonstrating the modification of Gilbert damping by the superconducting correlations are discussed.
\end{abstract}

DOI: 10.1103/PhysRevB.102.144521

\section{INTRODUCTION}

Spin transport and spin dynamics in superconductors have attracted significant attention recently [1-7]. Quite interesting experimental results have been obtained for the spin pumping effects, which, in general, play the central role in spintronics [8-10]. Ferromagnet/superconductor multilayers were found recently to demonstrate changes of the ferromagnetic resonance (FMR) frequency and linewidth [11-19] due to the superconducting correlations. Theoretical understanding of these effects is not complete yet. For example, a puzzling experimental result was obtained for the ferromagnetic insulator/superconductor multilayers where the pronounced peaks in the temperature dependence of Gilbert damping (GD) have been observed [16].

The enhancement of Gilbert damping due to the metal spin sink can be calculated using the linear response approximation [20], which involves the momentum $k$ and frequency $\Omega$ dependent spin susceptibility $\chi_{h}(k, \Omega)$ of the metal spin sink. Hence, to understand the modification of Gilbert damping due to the spin pumping in superconducting films it is necessary to calculate $\chi_{h}(k, \Omega)$ in the presence of spin relaxation mechanisms like the spin-orbit scattering.

Besides spin pumping phenomena the function $\chi_{h}(k, \Omega)$ is an important characteristic for other spin-dependent responses of superconductors. The local, that is, momentum-integrated, spin susceptibility determines the nuclear magnetic resonance [21] and the electronic paramagnetic resonance [22,23] relaxation rates. The coherence factor which arises due to the superconducting correlations provides the so-called HebelSlichter peak in the temperature dependence of the relaxation rates just below the critical temperature [21]. Besides that, the momentum-resolved spin susceptibility $\chi_{h}(k, \omega)$ was probed recently by inelastic neutron scattering [24]. The resonances in electron spin susceptibility measured by the neutronscattering response in the superconducting state have provided essential information to identify the gap function structure in iron pnictide compounds [25-28].

Despite the long history and currently renewed interest due to the applications in superconducting spintronics, the expression for spin susceptibility in superconductors is known only in the absence of spin relaxation mechanisms. The present work provides this expression for the usual spin-singlet $s$ wave superconductor. Equally important, a general approach for calculating spin susceptibility based on the solution of quasiclassical Eilenberger equations is developed.

Recent papers which have addressed this topic in connection with spin pumping $[29,30]$ reported finite zerotemperature dissipation at low frequencies: $\operatorname{Im}_{h}(q, \Omega) / \Omega \neq$ 0 at $\Omega \rightarrow 0$. This result contradicts physical intuition because there can be no dissipation at $\Omega<2 \Delta$ and in the absence of thermal quasiparticles which are frozen out in superconductors at $T \ll \Delta$, where $\Delta$ is the superconducting energy gap. As we show below this inconsistency comes from neglecting the important contributions while performing an analytical continuation procedure.

The first purpose of the present paper is to report the analytical expression for the finite-frequency spin susceptibility of superconductors with the spin-orbit relaxation mechanism. This result is a generalization of the classical work of Abrikosov and Gor'kov [31], who considered the static spin susceptibility to explain the finite Knight shift in superconductors at $T \ll \Delta$. We analyze several characteristic regimes, including large and strong spin relaxation, as well as the behavior for various values of the Dynes parameter [32].

The second purpose is to study the spin pumping in superconductor/ferromagnet systems by formulating the interfacial exchange model [20] in terms of the boundary 
conditions for quasiclassical Green's functions. The expressions for Gilbert damping are derived for the finite thickness of the spin sink layer. Also, we consider the system with an additional perfect spin absorber which can be realized experimentally by adding a layer of material with a very strong spin relaxation. The derived general expressions can be parametrized in terms of the dimensionless parameter characterizing the strength of the interfacial coupling between the ferromagnet and adjacent superconductor. Systems with elevated values of this parameter are predicted to feature a pronounced shift of the ferromagnetic resonance line induced by superconducting correlations.

\section{GENERAL FORMALISM}

\section{A. Diagrammatic formalism}

We describe the interaction of electrons with Zeeman field $\boldsymbol{h}=\boldsymbol{h}(\boldsymbol{r}, t)$ using the following Hamiltonian:

$$
\hat{V}_{P}=\hat{\boldsymbol{\sigma}} \boldsymbol{h},
$$

where $\hat{\sigma}=\left(\hat{\sigma}_{x}, \hat{\sigma}_{y}, \hat{\sigma}_{z}\right)$ is the vector of spin Pauli matrices. In addition, we assume the presence of disorder described by the Gaussian impurity potential. It has both the usual $u_{o}$ and the spin-orbit $u_{s o}$ scattering amplitudes:

$$
\hat{V}\left(\boldsymbol{p}, \boldsymbol{p}^{\prime}\right)=u_{o} \sum_{\boldsymbol{r}_{o}} e^{i \boldsymbol{r}_{o}\left(\boldsymbol{p}-\boldsymbol{p}^{\prime}\right)}+\frac{u_{s o}}{p_{F}^{2}} \hat{\boldsymbol{\sigma}} \cdot\left(\boldsymbol{p} \times \boldsymbol{p}^{\prime}\right) \sum_{\boldsymbol{r}_{s o}} e^{i \boldsymbol{r}_{s o}\left(\boldsymbol{p}-\boldsymbol{p}^{\prime}\right)},
$$

where $\boldsymbol{r}_{o}$ and $\boldsymbol{r}_{s o}$ denote the random impurity coordinates corresponding to the ordinary and spin-orbit scattering, respectively. We assume these coordinates are independent and thus neglect the magnetoelectric effects arising from the combined ordinary and spin-orbit scattering [33].

The spin polarization as a function of the imaginary time $t \in[0, \beta]$, where $\beta=1 / T$ is given by

$$
\boldsymbol{S}(t, \boldsymbol{r})=\frac{1}{4} \operatorname{Tr}[\hat{\boldsymbol{\sigma}} \hat{G}]\left(\boldsymbol{r}, \boldsymbol{r}, t_{1,2}=t\right),
$$

where $\hat{G}\left(\boldsymbol{r}_{1}, \boldsymbol{r}_{2}, t_{1,2}\right)$ is the imaginary-time Green's function (GF). The stationary propagators depend only on the relative time and coordinate. In the frequency and momentum representation they are given by $[31,34]$

$$
\begin{aligned}
\hat{G}_{0}(\omega, \boldsymbol{p}) & =\frac{\tilde{\Delta} \hat{\tau}_{2}-i \tilde{\omega} \hat{\tau}_{0}+\xi_{p} \hat{\tau}_{3}}{\tilde{\Delta}^{2}+\tilde{\omega}^{2}+\xi_{p}^{2}}, \\
\tilde{\omega} & =\omega \frac{\tilde{s}(\omega)}{s(\omega)}, \quad \tilde{\Delta}=\Delta \frac{\tilde{s}(\omega)}{s(\omega)},
\end{aligned}
$$

where $\xi_{p}=p^{2} / 2 m-\mu$ is the deviation of the kinetic energy from the chemical potential $\mu$ and $\hat{\tau}_{1,2,3}$ are the Pauli matrices in Nambu space. We denote $s=\sqrt{\omega^{2}+\Delta^{2}}$ and $\tilde{s}=s+1 / 2 \tau_{\text {imp }}$, where the scattering time is given by the superposition $\tau_{\text {imp }}^{-1}=\tau_{o}^{-1}+\tau_{s o}^{-1}$. We denote the usual $\tau_{o}^{-1}=$ $2 \pi n v u_{0}$ and spin-orbit $\tau_{s o}^{-1}=2 \pi n v u_{s o} / 3$ scattering rates. The propagator (4) is averaged over the randomly disordered point scatterer configurations.

We are interested in the spin polarization induced by the external Zeeman field $\boldsymbol{h}(t, \boldsymbol{r})=\boldsymbol{h}_{\Omega} e^{i \Omega t+i q r}$. The induced spin polarization as given by the diagram shown in Fig. 1(a) can be

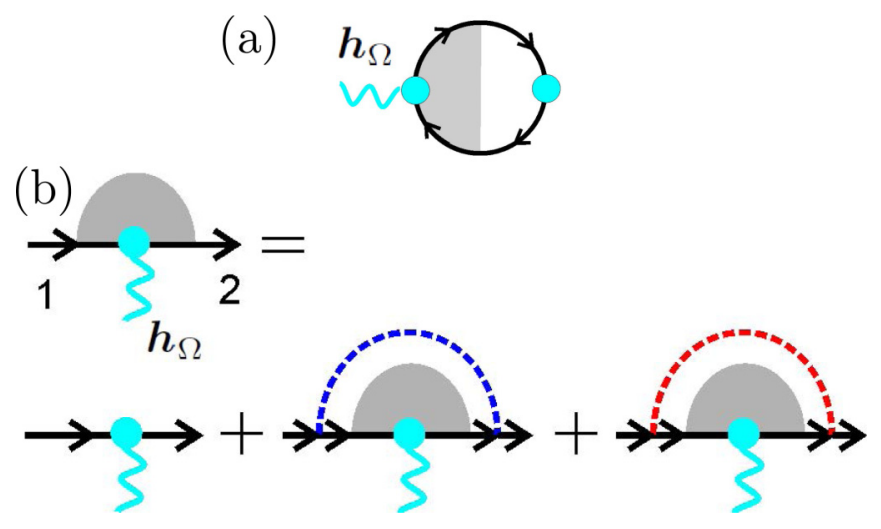

FIG. 1. (a) Bubble diagram for the linear response of spin polarization generated by the time-dependent Zeeman field $\boldsymbol{h}_{\Omega} e^{i \Omega t+i q r}$ shown by the wavy line. Circles show spin vertices $\hat{\sigma}$. The shaded region shows the impurity ladder. (b) Diagrammatic equation for the impurity ladder. The blue and red dashed lines correspond to the ordinary and spin-orbit scattering potentials averaged over the random impurity configuration.

written as follows:

$$
\boldsymbol{S}_{\Omega}=\chi_{h}(\Omega, \boldsymbol{q}) \boldsymbol{h}_{\Omega} .
$$

The linear spin susceptibility is defined by substituting into Eq. (3) $\hat{G}_{h}$, which is the first-order correction to the GF induced by the Zeeman field. The diagrammatic equation for this correction, which includes the summation of impurity ladder corrections, is shown Fig. 1(b). The shaded region denotes the impurity ladder corresponding to the ordinary and spinorbit impurity scatterings averaged over the random disorder configuration. The red and blue dashed lines correspond to the spin-orbit and ordinary impurity scattering potentials averaged over the randomly distributed impurities, respectively.

The analytical expression for the diagrammatic equation in Fig. 1(b) reads

$$
\begin{aligned}
\hat{G}_{h}(12)= & -\hat{G}_{0}(1) \hat{\boldsymbol{\sigma}} \boldsymbol{h}_{\Omega} \hat{G}_{0}(2)+\hat{G}_{0}(1) \frac{\hat{\boldsymbol{\sigma}}\left\langle\hat{g}_{h}\right\rangle \hat{\boldsymbol{\sigma}} \hat{\tau}_{3}}{6 i \tau_{s o}} \hat{G}_{0}(2) \\
& +\hat{G}_{0}(1) \frac{\left\langle\hat{g}_{h}\right\rangle \hat{\tau}_{3}}{2 i \tau_{o}} \hat{G}_{0}(2),
\end{aligned}
$$

where we have introduced the notation

$$
\hat{g}_{h}=\frac{i}{\pi} \int d \xi_{p} \hat{\tau}_{3} \hat{G}_{h}
$$

We use the condensed notation $\hat{G}_{0}(2)=\hat{G}_{0}(\omega, \boldsymbol{p})$ and $\hat{G}_{0}(1)=\hat{G}_{0}(\omega-\Omega, \boldsymbol{p}+\boldsymbol{q})$. The correction depends on the two frequencies and momenta $\hat{G}_{h}(12)=\hat{G}_{h}\left(\omega_{1}, \boldsymbol{p}, \omega_{2}, \boldsymbol{p}+\right.$ $\boldsymbol{q})$. The angular brackets denote the average over the momentum directions on the Fermi sphere so that in total $\left\langle\hat{g}_{h}\right\rangle=$ $(i / \pi v) \int d^{3} p \hat{G}_{h}$, where $v$ is the density of states at the Fermi level. Diagrammatically, the equation for the impurity ladder (7) is shown in Fig. 1(b). The second and third terms in Eq. (7), corresponding to the spin-orbit and ordinary scatterings, are shown by the blue and red dashed lines, respectively. As we will see below, the momentum-integrated correction $\hat{G}_{h}$ coincides with the solution of the quasiclassical Eilenberger equation [35] with collision integrals corresponding to the ordinary and spin-orbit scatterings [36]. 


\section{B. Quasiclassical formalism}

Under quite general conditions the nonequilibrium state of a metal involves perturbations of spectrum and distribution function in the vicinity of the Fermi level. For that the external fields should have frequencies much smaller than the Fermi energy and spatial scales much larger than the Fermi wavelength. Both these requirements are satisfied for the spin pumping systems. Hence, we can use the theory formulated in terms of the quasiclassical propagator [35]

$$
\hat{g}\left(\boldsymbol{r}, \boldsymbol{n}_{p}, t, t^{\prime}\right)=\frac{i}{\pi} \int d \xi_{p} \hat{\tau}_{3} \hat{G} .
$$

The calculation can be performed using either the imaginarytime formalism or the real-time formalism. In the imaginarytime domain the quasiclassical propagator is determined by the Eilenberger equation with collision integrals describing the impurity scattering [35]:

$$
\begin{aligned}
\left(\boldsymbol{v}_{F} \nabla\right) \hat{g}-i\left\{\hat{\tau}_{3} \partial_{t}, \hat{g}\right\}_{t} & =i\left[\hat{\tau}_{3} \hat{H}, \hat{g}\right]_{t}+\left[\left(\hat{\Sigma}_{o}+\hat{\Sigma}_{s o}\right) \circ, \hat{g}\right]_{t}, \\
\hat{\Sigma}_{s o} & =(\hat{\boldsymbol{\sigma}}\langle\hat{g}\rangle \hat{\boldsymbol{\sigma}}) / 6 \tau_{s o}, \\
\hat{\Sigma}_{o} & =\langle\hat{g}\rangle / 2 \tau_{o} .
\end{aligned}
$$

Here $\hat{\Sigma}_{o}$ and $\hat{\Sigma}_{s o}$ are the self-energies corresponding to the ordinary and spin-orbit scatterings, respectively [37], and $\hat{H}=\Delta \hat{\tau}_{2}+\hat{\boldsymbol{\sigma}} \boldsymbol{h}$. We denote the commutators $[X, g]_{t}=$ $X\left(t_{1}\right) g\left(t_{1}, t_{2}\right)-g\left(t_{1}, t_{2}\right) X\left(t_{2}\right)$ and the convolution $\langle\hat{g}\rangle \circ \hat{g}=$ $\int_{0}^{\beta} d t\langle\hat{g}\rangle\left(t_{1}, t\right) \hat{g}\left(t, t_{2}\right)$. The angle averaging over the Fermi surface is given by $\langle g\rangle$. The spin polarization is given by

$$
\boldsymbol{S}(t, \boldsymbol{r})=-i \frac{\pi v}{4} \operatorname{Tr}\left[\hat{\tau}_{3} \hat{\boldsymbol{\sigma}}\langle\hat{g}(t, t, \boldsymbol{r})\rangle\right] .
$$

The quasiclassical equations are supplemented by the normalization condition $\hat{g} \circ \hat{g}=1$.

\section{Analytical continuation}

In order to find the real-frequency response we need to implement the analytic continuation of Eq. (13). The firstorder correction to the quasiclassical GF can be written as $\hat{g}_{h}\left(t_{1}, t_{2}\right)=T \sum_{\omega} e^{-i \omega_{1} t_{1}+i \omega_{2} t_{2}} g\left(\omega_{1}, \omega_{2}\right)$, where $\omega_{2}=\omega$ and $\omega_{1}=\omega-\Omega$ are the fermionic Matsubara frequencies shifted by the Bosonic frequency $\Omega$ of the external Zeeman field. The analytic continuation of the sum is determined according to the general rule [38]

$$
\begin{aligned}
& T \sum_{\omega} g_{h}\left(\omega_{1}, \omega_{2}\right) \\
& \quad \rightarrow \int \frac{d \varepsilon}{4 \pi i} n_{0}\left(\varepsilon_{1}\right)\left[g_{h}\left(-i \varepsilon_{1}^{R},-i \varepsilon_{2}^{A}\right)-g_{h}\left(-i \varepsilon_{1}^{A},-i \varepsilon_{2}^{A}\right)\right] \\
& \quad+\int \frac{d \varepsilon}{4 \pi i} n_{0}\left(\varepsilon_{2}\right)\left[g_{h}\left(-i \varepsilon_{1}^{R},-i \varepsilon_{2}^{R}\right)-g_{h}\left(-i \varepsilon_{1}^{R},-i \varepsilon_{2}^{A}\right)\right],
\end{aligned}
$$

where $n_{0}(\varepsilon)=\tanh (\varepsilon / 2 T)$ is the equilibrium distribution function. On the right-hand side (rhs) of (14) we substitute $\varepsilon_{1}=\varepsilon-\Omega, \varepsilon_{2}=\varepsilon$ and $\varepsilon^{R}=\varepsilon+i \Gamma, \varepsilon^{A}=\varepsilon-i \Gamma$. Here the term with $\Gamma>0$ is added to shift the integration contour into the corresponding half plane. At the same time, $\Gamma$ can be used as the Dynes parameter [32] to describe the effect of different depairing mechanisms on spectral functions in the superconductor. We implement the analytical continuation in such a way that $s\left(-i \varepsilon^{R, A}\right)=-i \sqrt{\left(\varepsilon^{R, A}\right)^{2}-\Delta^{2}}$, assuming that the branch cuts run from $(\Delta, \infty)$ and $(-\infty,-\Delta)$.

The equilibrium GF in the imaginary-frequency domain is given by $\hat{g}_{0}(\omega)=\left(\hat{\tau}_{3} \omega+\hat{\tau}_{1} \Delta\right) / s(\omega)$. The real-frequency continuation reads $\hat{g}_{0}^{R, A}(\varepsilon)=\left(\hat{\tau}_{3} \varepsilon^{R, A}+i \hat{\tau}_{1} \Delta\right) / \sqrt{\left(\varepsilon^{R, A}\right)^{2}-\Delta^{2}}$. Thus, the linear response spin polarization is given by

$$
\begin{aligned}
\chi_{h}+1= & \int \frac{d \varepsilon}{4 \pi i} \chi\left(-i \varepsilon_{1}^{R},-i \varepsilon_{2}^{A}\right)\left[n_{0}\left(\varepsilon_{1}\right)-n_{0}\left(\varepsilon_{2}\right)\right] \\
& +\int \frac{d \varepsilon}{4 \pi i}\left[n_{0}\left(\varepsilon_{2}\right) \chi\left(-i \varepsilon_{1}^{R},-i \varepsilon_{2}^{R}\right)-n_{0}\left(\varepsilon_{1}\right)\right. \\
& \left.\times \chi\left(-i \varepsilon_{1}^{A},-i \varepsilon_{2}^{A}\right)\right],
\end{aligned}
$$

where we denote $\chi\left(\omega_{1}, \omega_{2}\right)=(\delta / \delta \boldsymbol{h}) \operatorname{Tr}\left[\sigma \hat{g}_{h}\left(\omega_{1}, \omega_{2}\right)\right]$. On the left-hand side (lhs) of Eq. (15) we subtract the off-shell contribution to the spin polarization due to the band edge shift by the Zeeman field.

It is interesting to note that in the superconducting state both the first and second terms on the rhs of (15) contribute to the dissipative part of spin susceptibility. With that we obtain the physically correct behavior in the low-temperature limit $\operatorname{Im} \chi_{h}(\Omega) / \Omega \rightarrow 0$ at $T \rightarrow 0$ and low frequency $\Omega \ll \Delta$. This is in contrast to previous calculations [29,30] which took into account only the first term in Eq. (15) and obtained physically incorrect finite dissipation in the absence of quasiparticles at $T=0$.

\section{SPIN SUSCEPTIBILITY}

\section{A. Diagram summation}

First, we demonstrate the connection between response functions determined by the diagram in Fig. 1(a) and by the solution of the time-dependent Eilenberger equation (10). Instead of using the usual approach of calculating the vertex function [31] we use the alternative route and solve directly the equation for the first-order correction Eq. (7). We use the general approach suggested recently [39] for deriving the equation for the momentum-integrated propagators $\hat{g}_{h}$ starting from the general equation for the exact GF (7).

The key idea of this derivation is based on the following trick. Let us multiply the function $\hat{G}_{h}(12)$ by $\hat{G}_{0}^{-1}(1)$ from the left and by $\hat{G}_{0}^{-1}(2)$ from the right, subtract the results, and integrate by $\xi_{p}$.

We use the fact that Eq. (4) yields the relations $\hat{G}_{0}^{-1}(j)=\tilde{\Delta}_{j} \hat{\tau}_{2}+i \tilde{\omega}_{j} \hat{\tau}_{0}+\xi_{p}\left(p_{j}\right) \hat{\tau}_{3}$ and $\tilde{\Delta}_{j} \hat{\tau}_{2}+i \tilde{\omega}_{j} \hat{\tau}_{0}=$ $i\left(s_{j}+1 / 2 \tau_{\text {imp }}\right) \hat{g}_{0}\left(\omega_{j}\right) \hat{\tau}_{3}$. Then we eliminate off-shell contributions in the momentum integrals to express the result through quasiclassical propagators,

$$
\begin{aligned}
& \int \frac{d \xi_{p}}{\pi}\left[\hat{G}_{0}^{-1}(1) \hat{G}_{h}-\hat{\tau}_{3} \hat{G}_{h} \hat{G}_{0}^{-1}(2) \hat{\tau}_{3}\right] \\
& =\tilde{s}_{1} \hat{g}_{0}(1) \hat{g}_{h}-\tilde{s}_{2} \hat{g}_{h} \hat{g}_{0}(2)-i\left(\boldsymbol{v}_{F} \boldsymbol{q}\right) \hat{g}_{h} .
\end{aligned}
$$

Next, let us derive the lhs of the equation for $\hat{g}_{h}$. Using the diagram in Fig. 1(b) or Eq. (7), we get

$$
\begin{aligned}
& \hat{G}_{0}^{-1}(1) \hat{G}_{h}-\hat{\tau}_{3} \hat{G}_{h} \hat{G}_{0}^{-1}(2) \hat{\tau}_{3} \\
& \quad=\hat{\tau}_{3} \hat{G}_{0}(1)\left(\hat{\boldsymbol{h}}_{\Omega}+i\left\langle\hat{g}_{h}\right\rangle \hat{\tau}_{3} / 2 \tau_{o}+i \boldsymbol{\sigma}\left\langle\hat{g}_{h}\right\rangle \boldsymbol{\sigma} \hat{\tau}_{3} / 6 \tau_{s o}\right) \hat{\tau}_{3} \\
& \quad-\left(\hat{\boldsymbol{h}}_{\Omega}+i\left\langle\hat{g}_{h}\right\rangle \hat{\tau}_{3} / 2 \tau_{o}+i \boldsymbol{\sigma}\left\langle\hat{g}_{h}\right\rangle \boldsymbol{\sigma} \hat{\tau}_{3} / 6 \tau_{s o}\right) \hat{G}_{0}(2),
\end{aligned}
$$


where we denote $\hat{\boldsymbol{h}}_{\Omega}=\hat{\boldsymbol{\sigma}} \boldsymbol{h}_{\Omega}$. Then combining Eqs. (16) and (17), we obtain the following equation with collision integrals $\hat{\mathcal{I}}_{o}$ and $\hat{\mathcal{I}}_{s o}$ :

$$
\begin{aligned}
& s_{1} \hat{g}_{0}(1) \hat{g}_{h}-s_{2} \hat{g}_{h} \hat{g}_{0}(2)-i\left(\boldsymbol{v}_{F} \boldsymbol{q}\right) \hat{g}_{h} \\
= & -i\left[\hat{g}_{0}(1) \hat{\boldsymbol{h}}_{\Omega} \hat{\tau}_{3}-\hat{\boldsymbol{h}}_{\Omega} \hat{\tau}_{3} \hat{g}_{0}(2)\right]+\hat{\mathcal{I}}_{s o}+\hat{\mathcal{I}}_{o}, \\
\hat{\mathcal{I}}_{o}= & {\left[\hat{g}_{0}(1)\left\langle\hat{g}_{h}\right\rangle+\left\langle\hat{g}_{h}\right\rangle \hat{g}_{0}(2)\right.} \\
& \left.-\left\langle\hat{g}_{h}\right\rangle \hat{g}_{0}(2)-\hat{g}_{0}(1) \hat{g}_{h}\right] / 2 \tau_{o}, \\
\hat{\mathcal{I}}_{s o}= & {\left[\hat{g}_{0}(1) \boldsymbol{\sigma}\left\langle\hat{g}_{h}\right\rangle \boldsymbol{\sigma}+3\left\langle\hat{g}_{h}\right\rangle \hat{g}_{0}(2)\right.} \\
& \left.-\boldsymbol{\sigma}\left\langle\hat{g}_{h}\right\rangle \boldsymbol{\sigma} \hat{g}_{0}(2)-3 \hat{g}_{0}(1)\left\langle\hat{g}_{h}\right\rangle\right] / 6 \tau_{s o} .
\end{aligned}
$$

Equation (18) coincides with the Eilenberger equation (10) expanded for the first-order correction $\hat{g}_{h}$. This proves that the time-dependent spin response in metals is captured by the Eilenberger equation with corresponding collision integrals.

\section{B. Susceptibility of the spatially homogeneous system}

First, we consider the spatially homogeneous system when the Zeeman field depends only on time and not on the spatial coordinate so that $\boldsymbol{q}=0$. The spatial dispersion of susceptibility is discussed in the diffusive limit in Sec. III C.

In the homogeneous case the ordinary scattering drops out from Eq. (18) since $\hat{\mathcal{I}}_{o}=0$. Then Eq. (18) can be solved analytically, yielding the frequency-resolved susceptibility $\chi(12)=(\delta / \delta \boldsymbol{h}) \operatorname{Tr}\left[\boldsymbol{\sigma} \hat{g}_{h}(12)\right]$ :

$$
\chi(12)=\left(\frac{\Delta^{2}-\omega_{1} \omega_{2}}{s_{1} s_{2}}+1\right) \frac{1}{s_{1}+s_{2}+4 / 3 \tau_{s o}},
$$

where $\omega$ are fermionic Matsubara frequencies, $\omega_{1}=\omega-\Omega$, $\omega_{2}=\omega, s_{1,2}=\sqrt{\omega_{1,2}^{2}+\Delta^{2}}$. Substituting this expression into the analytical continuation rule (15), we obtain the frequencydependent spin susceptibility $\chi_{h}=\chi_{h}(\Omega)$. It is interesting to note that this response function (21) is identical to the one which determines the finite-frequency conductivity of a superconductor. The first factor on the rhs of (21) is the usual coherence factor which arises due to the superconducting correlations in time-reversal symmetry-breaking responses [40]. It provides the peaked behavior of relaxation rates $\operatorname{Im} \chi_{h}(T)$ just below the critical temperature [21-23]. However, the second factor on the rhs of (21) can drastically change this behavior and even eliminate the coherence peak of $\operatorname{Im} \chi_{h}(T)$, as discussed below.

We can obtain analytical results in several important limiting cases. (i) For the normal metal $\Delta=0$ Eqs. (21) and (15) yield (see the detailed calculation in Appendix B)

$$
\chi_{h}(\Omega)=\frac{2\left(2 / 3 \tau_{s o}+\Gamma\right)}{2\left(2 / 3 \tau_{s o}+\Gamma\right)-i \Omega} .
$$

In this case the only contribution is provided by the first term in Eq. (15). As we can see, in the absence of spin relaxation $\Omega \tau_{s o} \rightarrow \infty$ and $\Gamma \rightarrow 0$ the susceptibility vanishes. Physically, this result is quite transparent because without relaxation the spin projection on the oscillating Zeeman field remains a good quantum number. Let us check that this result remains valid in the superconducting state. (ii) For that we consider the limit of the superconductor without spin relaxation. In this case using following relations $s_{1}^{2}-s_{2}^{2}=\omega_{1}^{2}-\omega_{2}^{2}$ and $2\left(\omega_{1} \omega_{2}-\Delta^{2}-s_{1} s_{2}\right)=\left(\omega_{1}+\omega_{2}\right)^{2}-\left(s_{1}+s_{2}\right)^{2}, \quad$ Eq. can be simplified as follows (see details in Appendix A):

$$
\chi(12)=\frac{2}{\Omega}\left(\frac{\omega_{1}}{s_{1}}-\frac{\omega_{2}}{s_{2}}\right) .
$$

Thus, making the analytical continuation and neglecting terms of the order of $\Gamma / \Omega$, we obtain

$$
\chi_{h}(\Omega)=-1-\int_{-\infty}^{\infty} \frac{d \varepsilon}{2 \Omega}\left[N\left(\varepsilon_{1}\right) n_{0}\left(\varepsilon_{1}\right)-N\left(\varepsilon_{2}\right) n_{0}\left(\varepsilon_{2}\right)\right],
$$

where $N(\varepsilon)$ is the normalized density of states (DOS), $\varepsilon_{1}=$ $\varepsilon-\Omega$, and $\varepsilon_{2}=\varepsilon$. We can see that this expression yields $\chi_{h}(\Omega)=0$ irrespective of the particular energy dependence of the DOS. This result can be qualitatively explained by the fact that in the absence of spin relaxation spin projection on the oscillating Zeeman field axis is a conserved quantity.

From this limiting case we can clearly see that to obtain the correct result it is necessary to take into account all parts in Eq. (15). Indeed, the contribution of the first term in Eq. (15) is proportional to $\int d \varepsilon\left[\hat{g}_{0}^{R}(1)-\hat{g}_{0}^{A}(2)\right] \partial_{\varepsilon} n_{0} \approx 2 \Omega / \Delta$ at low temperatures. This contribution is canceled by the second term in Eq. (14), yielding $\chi_{h}(\Omega)=0$ for $\tau_{s o}^{-1}=0$.

As we have obtained in the normal metal limit, the contribution of the first term in spin susceptibility (15) is of the order of $\Omega \tau_{s}$ for weak spin relaxation $\Omega \tau_{s} \gg 1$. Thus, when $\tau_{s o} \Delta \gg$ 1 , the contribution of the second term can be neglected. For stronger spin-orbit relaxation such an approximation as used in previous works $[29,30]$ is inaccurate. Below we confirm this conclusion by evaluating Eq. (15) numerically.

Let us now considered the opposite limit of (iii) a superconductor with strong spin relaxation $\tau_{s o} \Delta \ll 1$ and low frequencies $\Omega \ll \Delta$. In this case from the general equation (21) we obtain

$$
\chi(12)=\frac{3 \tau_{s o}}{4}\left(\frac{\Delta^{2}-\omega_{1} \omega_{2}}{s_{1} s_{2}}+1\right) .
$$

Substituting this expression into the analytical continuation rule (15), after some algebra, we get

$$
\frac{4}{3 \tau_{s o}} \frac{\operatorname{Im} \chi_{h}}{\Omega}=\int_{-\infty}^{\infty} d \varepsilon\left(\Delta^{2} / \varepsilon^{2}+1\right) N^{2} \partial_{\varepsilon} n_{0}
$$

From this expression we can see analytically that the dissipative part of the susceptibility vanishes in the zero-temperature limit.

General case. Now let us consider the behavior of spin susceptibility in the wide range of parameters by evaluating numerically the integral in Eq. (15). First, we compare the results given by the full Eq. (15) with the contribution of only the first term. The sequence of plots in Fig. 2 shows the temperature dependence of the dissipative part $\operatorname{Im} \chi_{h}$ at $\Omega=0.01 T_{c}$, Dynes parameter $\Gamma=0.001 T_{c}$, and several values of the spin-orbit scattering rate. The dependencies given by the full Eq. (15) are shown by the blue solid curves, while the dependencies given only by the first term in Eq. (15) are shown by the red dashed curves. We can see that for weak spin-orbit scattering $\tau_{s o} T_{c} \gg 1$ these curves coincide, according to the conclusion we have made based on the analysis of the limiting cases above. However, there is a large discrepancy 

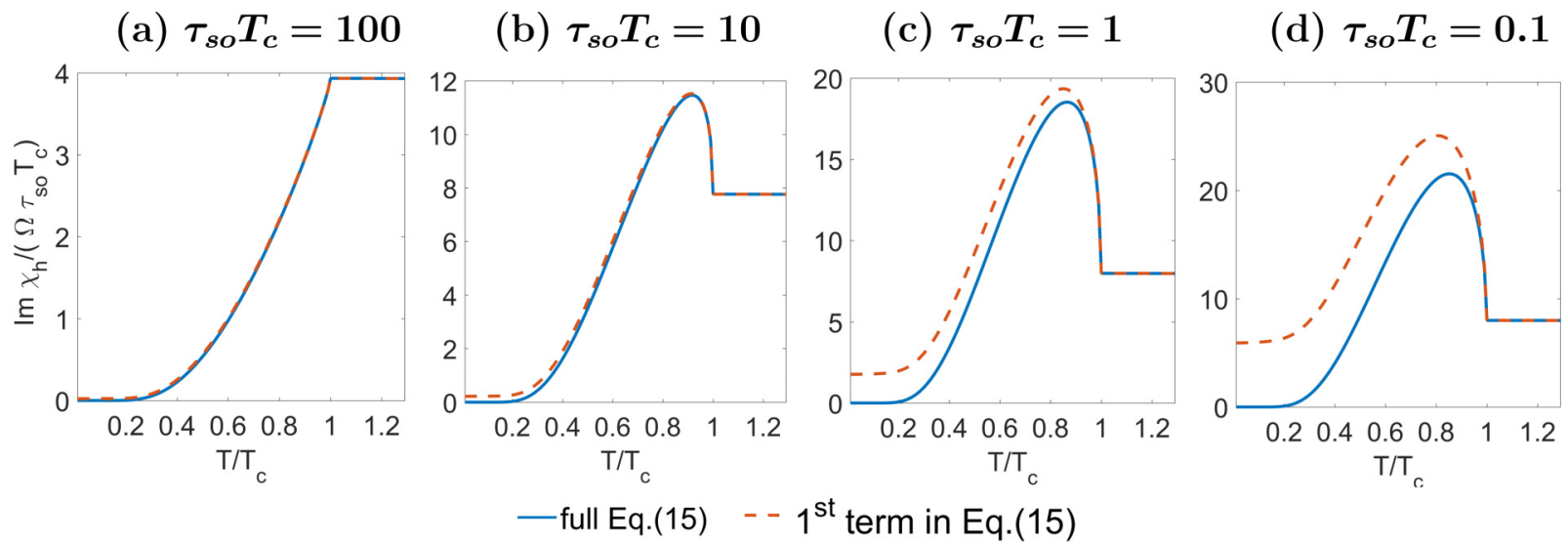

FIG. 2. Comparison of the contributions to the dissipative spin response $\operatorname{Im} \chi_{h}$ given by both terms in Eq. (15) (blue solid lines) and only the first term in Eq. (15) (red dashed lines). The parameters are $\Gamma=0.001 T_{c}, \Omega=0.01 T_{c}$, and spin-orbit scattering time $\tau_{s o} T_{c}$ is (a) 100 , (b) 10 , (c) 1 , and (d) 0.1 .

for stronger spin-orbit relaxation $\tau_{s o} T_{c}<1$. Note that the behavior of the dashed curves is similar to that obtained for the dissipation signal in previous works [29]. That is, at $\tau_{s o} T_{c}<1$ they significantly deviate from zero at $T \rightarrow 0$. As we have noted, the finite value of $\operatorname{Im} \chi_{h}$ in the low-temperature limit is physically incorrect. On the other hand, the solid curves always demonstrate the correct behavior going to zero in the limit $T \rightarrow 0$. Thus, the numerical analysis also confirms that both terms in Eq. (15) contribute to the dissipative part of the spin response in the superconducting state.

Next, let us consider how the temperature dependencies of $\operatorname{Im} \chi_{h}$ at $\Omega=0.01 T_{c}$ change with the Dynes parameter. The sequence of plots for the three values of $\Gamma / T_{c}=$ $0.001,0.01,1$ is shown in Fig. 3 for different values of the spin-orbit relaxation rate. One feature demonstrated by these curves is that the peak in the temperature dependencies becomes less pronounced and disappears for weak spin relaxation. At the same time the relative height of the peak almost does not change between strong $\left[\tau_{s o} T_{c}=1 ;\right.$ Fig. 3(c) $]$ and very strong $\left[\tau_{s o} T_{c}=0.1\right.$; Fig. 3(d)] spin relaxation.

Besides that, we can see that the height of the peak is strongly suppressed by increasing the Dynes parameter. For the realistic value in the superconductor $\mathrm{NbN} \Gamma=0.1 T_{c}$ the relative height of the peak is about $0.2-0.5$ of the normal metal value at $T>T_{c}$. This increase is, by an order of magnitude, weaker than the relative peak heights of 2-3 observed in the spin pumping experiment in $\mathrm{GdN} / \mathrm{NbN}$ bilayers [16]. Therefore, we can assume that there should be a different explanation for this experiment other than the peaked behavior of spin susceptibility [29].

Now let us consider the behavior of the spin susceptibility at larger frequencies comparable with superconducting energy scales $\Omega \sim T_{c}$. In this case it is interesting to consider both the dissipative and nondissipative parts of spin susceptibility. As we show below, they are responsible for the damping and fieldlike spin torque contributions to the spin dynamics. In Fig. 5 we plot the relevant quantities $\operatorname{Im} \chi_{h}(\Omega) / \Omega$, which contributes to the excess Gilbert damping, and $\operatorname{Re} \chi_{h}(\Omega)-\operatorname{Re} \chi_{h}(0)$, which contributes to the shift of the ferromagnetic resonance central frequency. First, we notice that the nonmonotonic temperature dependence of the dissipative part (left panels in Fig. 5) disappears at frequencies much larger than the Dynes parameter $\Omega \gg \Gamma$. For such frequencies $\operatorname{Im} \chi_{h}$ monotonically decreases with temperature and finally disappears at $T \rightarrow 0$, provided that $\Omega<2 \Delta$. For $\Omega>2 \Delta$ there is a nonzero signal even at
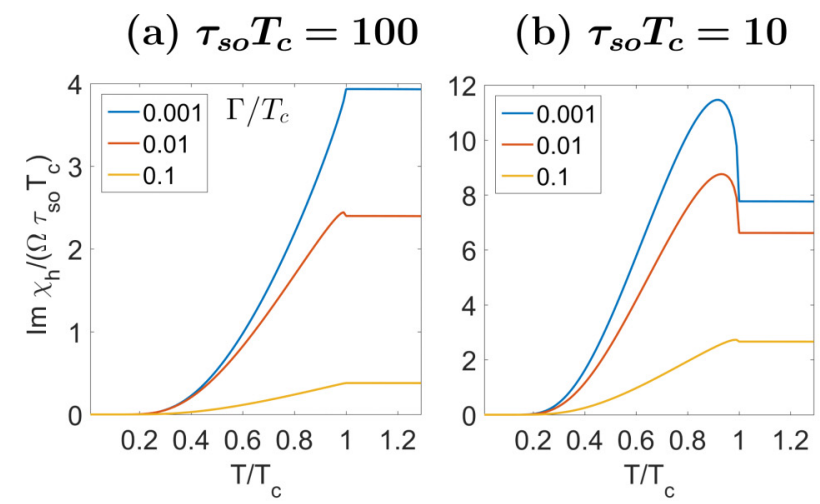

(c) $\tau_{s o} T_{c}=1$

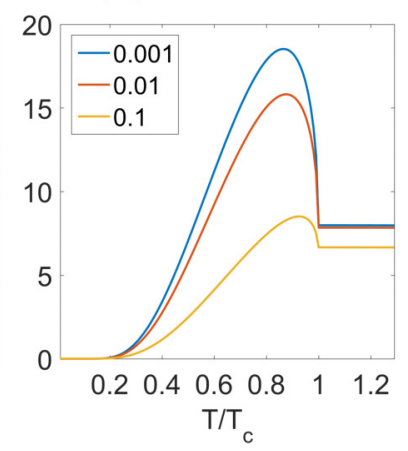

(d) $\tau_{s o} T_{c}=0.1$

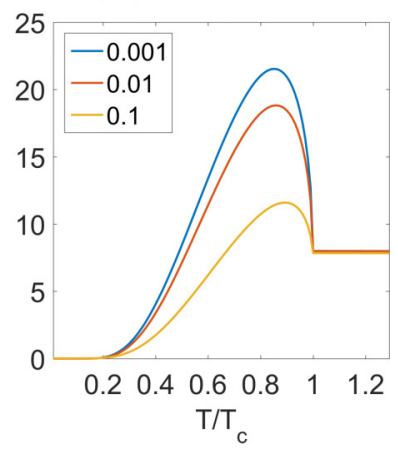

FIG. 3. Temperature dependencies of the dissipative part of the spin susceptibility $\operatorname{Im} \chi_{h}$ at small frequency $\Omega=0.01 T_{c}$. In each panel curves from top to bottom correspond to the Dynes parameter values $\Gamma / T_{c}=0.001,0.01,0.1$. The spin-orbit scattering time $\tau_{s o} T_{c}$ is (a) 100 , (b) 10 , (c) 1 , and (d) 0.1 . 
$T=0$ due to the excitation of quasiparticles across the gap.

\section{Spatial dispersion of the susceptibility}

In general, due to the presence of the anisotropic term in Eq. (18) the analytical solution is not possible for $\boldsymbol{q} \neq 0$. However, we can still get the analytical solution in the experimentally relevant diffusive limit when the ordinary scattering rate is very large, $\left(T_{c} \tau_{o}\right)^{-1} \gg 1$. In this case Eq. (10) can be simplified by averaging over momentum directions. The isotropic part of the GF satisfies the Keldysh-Usadel equation

$$
-i\left\{\hat{\tau}_{3} \partial_{t}, \check{g}\right\}_{t}+D \nabla(\check{g} \circ \nabla \check{g})=i\left[\hat{\tau}_{3} \hat{H}, \check{g}\right]_{t}+\left[\check{\Sigma}_{s o} \circ, \check{g}\right]_{t},
$$

where $D=\tau_{o} v_{F}^{2} / 3$ is the diffusion coefficient.

The spin response to the spatially inhomogeneous Zeeman field $\boldsymbol{h}_{\Omega} e^{i \Omega t+i q z}$ can be calculated analytically in the diffusive limit using the Usadel equation (27). Using the imaginarytime representation and searching for the solution in the form $\hat{g}_{h}(12) e^{-i q z} e^{i\left(\omega_{1} t_{1}-\omega_{2} t_{2}\right)}$, we obtain the linearized Usadel equation

$$
\begin{aligned}
& \left(s_{1}+D q^{2}\right) \hat{g}_{0}(1) \hat{g}_{h}-s_{2} \hat{g}_{h} \hat{g}_{0}(2) \\
& \quad=i\left(\boldsymbol{h}_{\Omega} \hat{\boldsymbol{\sigma}}\right)\left[\hat{g}_{0}(1) \hat{\tau}_{3}-\hat{\tau}_{3} \hat{g}_{0}(2)\right] .
\end{aligned}
$$

The solution of this equation yields susceptibility in the form of (21) with the substitution of the effective spin relaxation time $4 / 3 \tau_{s o} \rightarrow 4 / 3 \tau_{s o}+D q^{2}$,

$$
\chi(12)=\frac{\Delta^{2}+s_{1} s_{2}-\omega_{1} \omega_{2}}{s_{1} s_{2}\left(s_{1}+s_{2}+D q^{2}+4 / 3 \tau_{s o}\right)} .
$$

This expression together with Eq. (15) can be used to study various phenomena related to the spin dynamics in superconductors with spin-orbit relaxation. For example, it is possible to study the effect of spin relaxation on the nuclear magnetic resonance [21,41] and electron paramagnetic resonance [23] in superconductors. It is interesting that the peak in spin relaxation observed in these experiments is robust against even the very strong spin-orbit scattering, which follows from Figs. 3(d) and 4. In the limit of weak spin relaxation there is no peak; that is, the temperature dependence is monotonous, as shown in Figs. 2(a) and 3(a).

\section{Keldysh formalism and kinetic equations}

In the general case the procedure of analytical continuation is not possible, and one has to consider the real-time equations from the very start. This brings extra complication related to the matrix structure of the contour-ordered propagator

$$
\check{g}=\left(\begin{array}{cc}
\hat{g}^{R} & \hat{g}^{K} \\
0 & \hat{g}^{A}
\end{array}\right)
$$

having the spectral retarded (advanced) $\hat{g}^{R(A)}$ and the Keldysh component $\hat{g}^{K}$.

The matrix GF satisfies the Keldysh-Usadel equation, which is formally identical to Eqs. (10) or (27) with the substitution $\partial_{t} \rightarrow-i \partial_{t}$. Using the normalization condition $\hat{g}^{R} \circ \hat{g}^{K}+\hat{g}^{K} \circ \hat{g}^{A}=0$, we can introduce the parametrization of the Keldysh component in terms of the distribution function (a) $\Gamma=0.1 T_{c}$

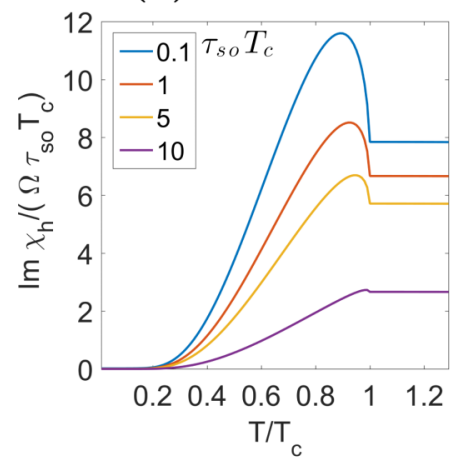

(b) $\Gamma=0.01 T_{c}$

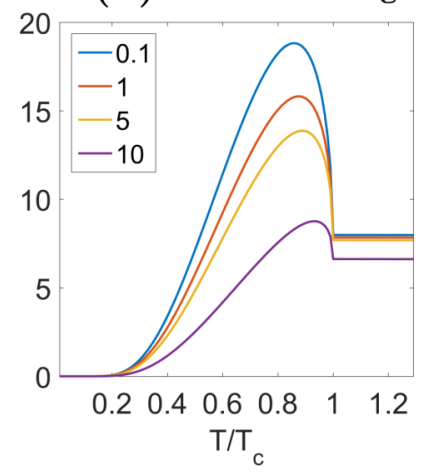

FIG. 4. Temperature dependencies of the dissipative part of the spin susceptibility at $\Omega=0.01 T_{c}$ and different values of the Dynes parameter: (a) $\Gamma=0.1 T_{c}$ and (b) $\Gamma=0.01 T_{c}$. Curves from top to bottom in each panel correspond to the spin-orbit scattering times $\tau_{s o} T_{c}=0.1,1,5,10$.

$\hat{g}^{K}=\hat{g}^{R} \circ \hat{f}-\hat{f} \circ \hat{g}^{A}$. Local spin density is given by

$$
\boldsymbol{S}(t)=-\frac{\pi v}{4} \operatorname{Tr}\left[\hat{\boldsymbol{\sigma}} \hat{\tau}_{3} \hat{g}^{K}(t, t)\right] .
$$

The driven state of the superconductor is described by the deviation of the Keldysh function from equilibrium, which consists of the parts with perturbations of spectral functions $\delta \hat{g}^{R, A}$ and the nonequilibrium part of distribution function $\delta \hat{f}$.

(a) $\Gamma=0.1 T_{c}$
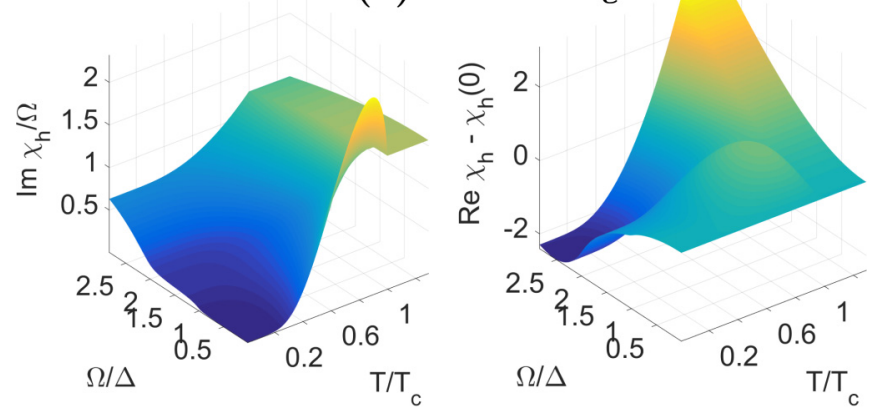

(b) $\Gamma=0.001 T_{c}$
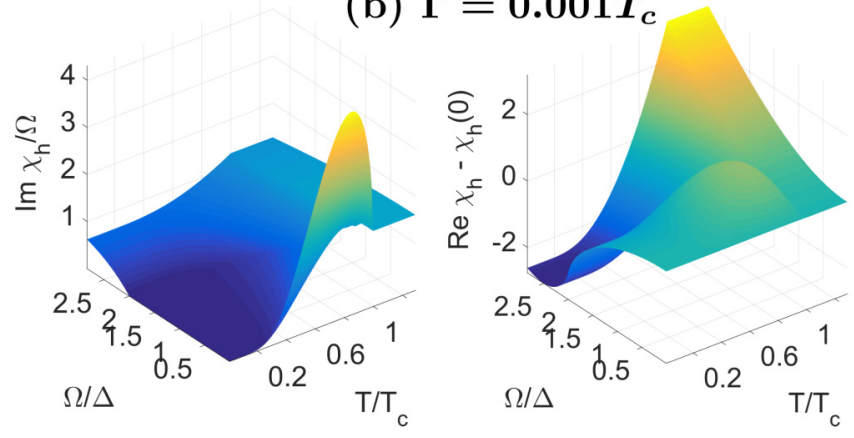

FIG. 5. Imaginary (left column) and real (right column) parts of the spin susceptibility as functions of $T$ and $\Omega$, normalized to the zero-temperature gap $\Delta(T=0)$. The Dynes parameters are (a) $\Gamma=0.1 T_{c}$ and (b) $\Gamma=0.001 T_{c}$. The spin-orbit scattering time is $\tau_{s o} T_{c}=1$. 
In the linear response regime we can write

$$
\begin{aligned}
\delta \hat{g}^{K}(12)= & {\left[\hat{g}_{0}^{R}(1)-\hat{g}_{0}^{A}(2)\right] \delta \hat{f}+\delta g^{R}(12) n_{0}(2) } \\
& -\delta g^{A}(12) n_{0}(1) .
\end{aligned}
$$

Comparing expressions (31) and (30) with (15), we can see that the first term here yields the first term on the rhs of Eq. (15) and $\hat{f} \propto n\left(\varepsilon_{1}\right)-n\left(\varepsilon_{2}\right)$.

In the low-frequency limit we can calculate the corrections to the distribution function using the kinetic equation [2] with the driving term obtained from the gradient expansion of the mixed product in the analytical continuation of Eq. (27),

$$
[\hat{H}, \hat{f}]_{t}=i \hat{\boldsymbol{\sigma}} \partial_{t} \boldsymbol{h} \partial_{\varepsilon} n_{0} .
$$

Parametrizing the spin-dependent distribution function as $\hat{f}=\hat{\sigma} \boldsymbol{f}$, we get the kinetic equation, which for the spatially homogeneous system is given by

$$
\begin{gathered}
\partial_{t} \boldsymbol{f}+\left(2 \Gamma+\tau_{s}^{-1}\right) \boldsymbol{f}=\partial_{\varepsilon} n_{0} \partial_{t} \boldsymbol{h}, \\
\tau_{s}^{-1}=\left(1 / 3 \tau_{s o}\right) N^{-1} \operatorname{Tr}\left(1-\hat{g}^{R} \hat{g}^{A}\right),
\end{gathered}
$$

where $N=\operatorname{Re} \operatorname{Tr}\left[\hat{\tau}_{3} \hat{g}^{R}\right] / 2$ is the normalized density of states.

At the subgap energies $|\varepsilon|<\Delta$ the spin relaxation rate (34) is not defined if the density of states is strictly zero, $N=0$. However, for the finite Dynes parameter $N \propto \Gamma$, so that $\tau_{s}^{-1} \propto$ $\Gamma$. The solution of Eq. (33) yields the contribution to the spin density,

$$
\chi_{\text {kin }}+1=\frac{\Omega}{2} \int_{-\infty}^{\infty} d \varepsilon \frac{N \partial_{\varepsilon} n_{0}}{\Omega-i\left(2 \Gamma+\tau_{s}^{-1}\right)},
$$

which coincides with the first term in Eq. (15) in the lowfrequency limit.

\section{SPIN PUMPING IN SUPERCONDUCTING FILMS}

With the general expression for spin susceptibility in hand we can study the effects of spin pumping from the ferromagnet into the adjacent metallic film. The schematic setups are shown in Fig. 6. The metallic spin sink (M) has an interface with (a) vacuum and (b) the perfect spin absorber. The corresponding boundary conditions are (a) vanishing spin current and (b) vanishing nonequilibrium spin polarization at $z=d_{M}$.

To quantify the spin pumping effect we consider the interfacial exchange interaction between the localized spins in the ferromagnetic film (F) and conduction elections in $\mathrm{M}$. Within this model the local spin polarization close to the interface $\boldsymbol{S}(t)$ acts as an effective field for the localized magnetic moments. This process can be taken into account by introducing the additional term $\boldsymbol{i}(t)$ into the Landau-Lifshitz-Gilbert (LLG) equation

$$
\begin{gathered}
(1+\alpha \boldsymbol{m} \times) \partial_{t} \boldsymbol{m}+\gamma \boldsymbol{m} \times \boldsymbol{H}_{\mathrm{eff}}=\boldsymbol{i} / S_{F 0} d_{F}, \\
\boldsymbol{i}(t)=J_{s d} \boldsymbol{S}(t) \times \boldsymbol{m}(t) .
\end{gathered}
$$

Here $S_{F 0}$ is the equilibrium spin density in $\mathrm{F}, d_{F}$ is the $\mathrm{F}$ film thickness, $\boldsymbol{H}_{\text {eff }}$ is the effective field, and $\alpha$ is the intrinsic Gilbert damping coefficient. The term $\boldsymbol{i}(t)$ can be interpreted as the spin current between $\mathrm{F}$ and $\mathrm{M}$.

To calculate $S(t)$ we use the spin susceptibility (6) with the effective Zeeman field determined by the spin-dependent scat- (a)

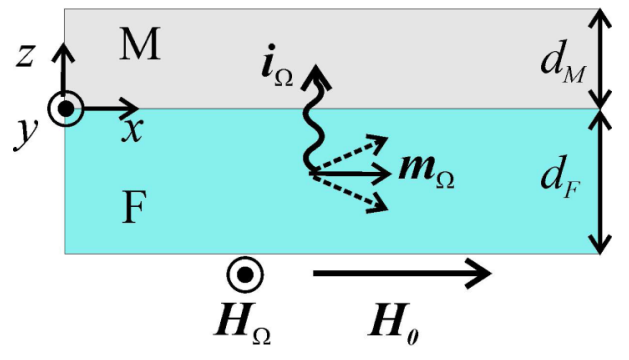

(b)

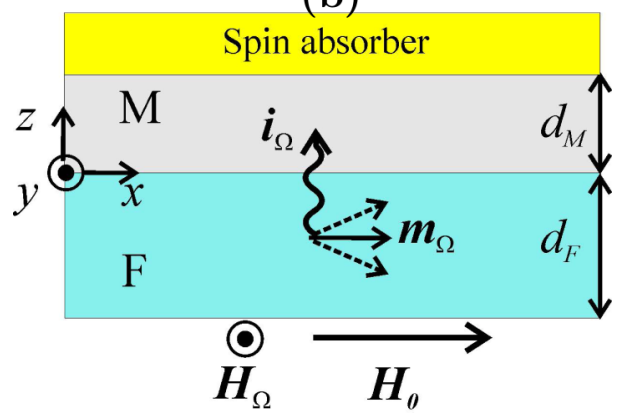

FIG. 6. Schematic setup with the interface between the metallic spin sink (M) and the ferromagnetic film (F) with widths $d_{M}$ and $d_{F}$, respectively. The constant external magnetic field is $H_{0} x$. The magnetization precession $\boldsymbol{m}_{\Omega} e^{i \Omega t}$ is driven by the external magnetic field $H_{\Omega} e^{i \Omega t} \boldsymbol{y}$. It generates spin current $\boldsymbol{i}_{\Omega}$ pumped from $\mathrm{F}$ to M. (a) M has an interface with vacuum; (b) $\mathrm{M}$ has an interface with the perfect spin absorber.

tering at the $\mathrm{F}$ interface. The linear response regime explored below is, in general, valid if $h_{\text {eff }} \ll \tanh \left(d_{M} / l_{s o}\right)\left(D / l_{s o} d_{M}\right)$, where the effective exchange field $h_{\text {eff }}=J_{s d} / d_{M}$ and $l_{s o}=$ $\sqrt{D \tau_{s o}}$ is the spin relaxation length. In the linear regime the local spin polarization near the $\mathrm{F}$ interface can be written as follows:

$$
\begin{gathered}
\boldsymbol{S}_{\Omega}=v h_{\mathrm{eff}} \chi_{m} \boldsymbol{m}_{\Omega}, \\
\chi_{m}(\Omega)=\sum_{n=0}^{\infty} \chi_{h}\left(q_{n}, \Omega\right),
\end{gathered}
$$

where we introduce the local spin susceptibility $\chi_{m}$ which determines the response to the $\delta$-functional Zeeman field. The summation in Eq. (39) runs over the discrete set of momenta given by $q_{n}=n \pi / d_{M}$ for the vacuum interface [Fig. 6(a)] which is determined by the zero boundary condition for the spin current at the interface with vacuum $z=d_{M}$. For the strong spin sink interface [Fig. 6(b)] we have $q_{n}=(n+$ $1 / 2) \pi / d_{M}$, which is determined by the zero boundary of the nonequilibrium spin polarization which is suppressed by the strong spin sink at $z=d_{M}$. The derivation of this result is given in Appendix D.

To calculate the particular values of the GD we can use the spin susceptibility in the presence of spin-orbital scattering (29). In principle, the presence of a spin-active $F$ interface should also lead to spin-flip scattering similar to the effect of magnetic impurities. Although it is possible to take this effect into account, we neglect it in the present study 
because it does not lead to qualitative changes in the Gilbert damping behavior. Likewise, we neglect the effect of residual electron-electron interactions on the spin susceptibility. The effect of interactions in metals is usually determined by the Fermi-liquid corrections, which are assumed to be small. This approximation breaks, e.g., for systems which are close to magnetic instabilities or have Fermi surfaces with nesting behavior such as iron pnictide compounds [25,26].

Taking into account Eq. (29), we can see that the only difference introduced by the spin absorber [Fig. 6(b)] is the modification of the spin relaxation rate to $\tau_{s o}^{-1} \rightarrow \tau_{s o}^{-1}+$ $D\left(\pi / 2 d_{M}\right)^{2}$. Therefore, hereafter, we will not distinguish between these two cases, implying that the effective spin relaxation is used.

The Fourier components of the spin current (37) are given by

$$
\boldsymbol{i}(\Omega)=v h_{\mathrm{eff}}^{2} d_{M}\left[\chi_{m}(\Omega)-\chi_{m}(0)\right] \boldsymbol{m} \times \boldsymbol{m}_{\Omega} .
$$

For the configuration in Fig. 6 the effective field is given by $\boldsymbol{H}_{\text {eff }}=H_{\Omega} e^{i \Omega t} \boldsymbol{y}+B_{0} \boldsymbol{x}$, where $B_{0}=H_{0}+4 \pi M$. In this case the eigenfrequencies of the LLG equation (36) satisfy the equation

$$
\begin{gathered}
\Omega=\sqrt{\left(\gamma B_{0}+\delta \omega\right)\left(\gamma H_{0}+\delta \omega\right)}, \\
\delta \omega=i \Omega \alpha+\left[\chi_{m}(\Omega)-\chi_{m}(0)\right] T_{c} C, \\
C=\frac{h_{\mathrm{eff}}}{T_{c}} \frac{v h_{\mathrm{eff}}}{S_{F 0}} \frac{d_{M}}{d_{F}},
\end{gathered}
$$

The extra dissipation, that is, the imaginary part of $\Omega$ in Eq. (41), can be considered to result from the effective Gilbert damping constant increase,

$$
\delta \alpha=C T_{c} \operatorname{Im} \chi_{m} / \Omega
$$

In the case where the film thickness is small, $d_{M}<$ $\min \left(l_{s o}, \xi\right)$, where $\xi=\sqrt{D / T_{c}}$ is the zero-temperature coherence length, only the contribution with $n=0$ in the sum (39) is important. In this case the spin pumping effect is totally determined by the homogeneous spin-orbit relaxation, so that $\chi_{m}(\Omega) \approx \chi_{h}(\Omega, q=0)$.

For a larger film thickness we need to take into account several terms in Eq. (39). Only for the very large thickness, $d_{M} \gg \min \left(l_{s o}, \xi\right)$, is the expression used in previous works $[20,29]$ recovered in the form

$$
\delta \alpha=\frac{\nu J_{s d}^{2}}{d_{F} S_{F 0}} \int_{-\infty}^{\infty} \frac{d q}{\pi} \frac{\operatorname{Im} \chi_{h}(q, \Omega)}{\Omega} .
$$

Temperature dependencies of the normalized excess Gilbert damping are shown in Fig. 5. We can see that these dependencies are qualitatively similar to that obtained in the absence of spin relaxation for the superconducting film of a very large thickness [42]. They are also qualitatively similar to the temperature dependencies of the NMR [21,41] and EPR $[22,43]$ linewidths in superconductors. Note that for a relatively large Dynes parameter $\Gamma=0.1 T_{c}$ the peak in the temperature dependencies of the Gilbert damping is almost absent (red curves in Fig. 7), and superconductivity leads to the monotonous suppression of the spin pumping dissipative signal. This result reproduces theoretically the behavior observed in FMR experiments with $\mathrm{Py} / \mathrm{Nb}$ bilayers [11]. Using
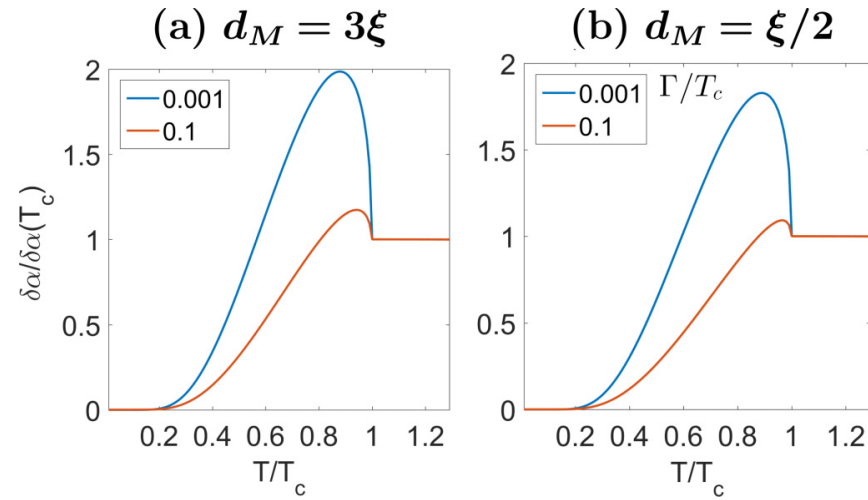

FIG. 7. Temperature dependencies of the additional Gilbert damping coefficient $\delta \alpha$ [Eq. (44)] at low frequency $\Omega=0.01 T_{c}$. In each panel curves corresponding to the Dynes parameter values $\Gamma / T_{c}=0.001,0.1$ are shown. The spin-orbit scattering time $\tau_{s o} T_{c}=$ 4 , corresponding to the normal state spin relaxation length $l_{s o}=\xi / 2$. The metallic film thickness is (a) $d_{M}=3 \xi$ and (b) $d_{M}=0.5 \xi$.

a large Dynes parameter $\Gamma \sim T_{c}$, we can describe qualitatively the effect of superconducting gap suppression near the surface of the metallic ferromagnet such as $\mathrm{Fe}$ or Ni. At the same time the Dynes parameter $\Gamma=0.1 T_{c}$ corresponds to superconductors with a large electron-phonon relaxation rate such as $\mathrm{NbN}$. Therefore, provided the mechanism of spin pumping between the FI and $\mathrm{NbN}$ superconductor is correctly described by Eqs. (45) or (44), the Gilbert damping behavior should correspond to the red curves in Fig. 7 with rather weak peaks. The amplitude of these peaks is much smaller than has been observed in the experiment [16]. This discrepancy shows the presence of some other yet unknown mechanism of spin pumping which can yield more pronounced peaks. The identification of such a mechanism is, however, beyond the scope of the present paper.

Quite interestingly, spin relaxation and superconducting correlations lead to the pronounced frequency dependence of the real part of the susceptibility $\operatorname{Re} \chi_{h}$, as shown in the right panels of Fig. 5. This leads to the additional contribution to the spin pumping having the form of the fieldlike spin torque, which is an additional frequency-dependent effective field acting on the magnetization of the ferromagnet $\boldsymbol{m}$. This leads to the shift of the FMR central frequency, which can be obtained from Eq. (41) as follows:

$$
\delta \Omega=C T_{c} \frac{\operatorname{Re}\left[\chi_{m}(\Omega)-\chi_{m}(0)\right]}{2 \Omega} \gamma\left(B_{0}+H_{0}\right)
$$

This shift is negligible at small frequencies $\Omega T_{c} \ll 1$ and $\Omega \tau_{s o} \ll 1$ and small interfacial coupling between $\mathrm{F}$ and M films measured by the dimensionless parameter (43). However, it becomes significant for higher frequencies and larger $C$.

To quantify the superconductivity-induced FMR frequency shift we consider the system with not very strong spin relaxation $\tau_{s o} T_{c}=1$. The normalized FMR response function, according to Eqs. (36) and (41), is proportional to $\left[\Omega^{2}-\right.$ $\left.\left(\gamma B_{0}+\delta \omega\right)\left(\gamma H_{0}+\delta \omega\right)\right]^{-1}$. In Fig. 8 we normalize this response function to its largest value at each temperature so that 

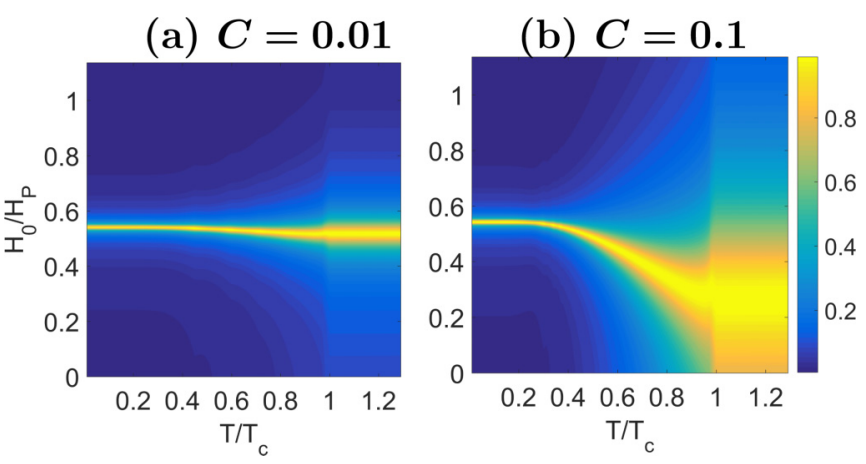

FIG. 8. Normalized amplitude of the FMR response signal as a function of the constant external magnetic field $H_{0}$ and temperature $T$. The magnetic field is measured in the units $H_{p}=\Delta(T=0) / \gamma$. The spin relaxation time is $\tau_{s o} T_{c}=1$, and the frequency is $\Omega=T_{c}$. We consider (a) weak $C=0.01$ and (b) relatively large $C=0.1$ values of the interfacial coupling parameter (43).

it is possible to see the transformation of the FMR line in the superconducting state.

We can see two pronounced effects which appear with an increase in the coupling parameter. First, comparing Figs. 8(a) and 8(b) at $T>T_{c}$, we can see a significant growth of the normal state resonance linewidth. Given the fact that in the experiment [16] with FMR in ferromagnetic insulator/superconductor multilayers the resonance is well defined at $\Omega \approx 0.01 T_{c}$, we can conclude that the coupling parameter is $C \sim 0.01$, corresponding to Fig. 8(a). In this case there is no noticeable shift of the FMR resonance line as a function of temperature.

From its definition (43), the coupling parameter $C \propto$ $\left(d_{F} d_{M}\right)^{-1}$ can be increased by decreasing the thickness of either the metal film $d_{M}$ or the ferromagnetic film $d_{F}$. By doing so and reaching a value of $C=0.1$, one would be able to see that the superconducting correlations produce significant shift of the temperature dependence of the resonant field $H_{0}$.

\section{CONCLUSION}

We have derived and analyzed the general expression for the time-dependent linear spin response in the superconductor with spin-orbit relaxation. The homogeneous spin susceptibility is found for any amount of the ordinary disorder. In the spatially inhomogeneous case the diffusive limit is considered. We show that the effective spin relaxation rate is given by the sum of the spin-orbit scattering rate and the diffusive term.

At low frequencies, $\Omega \ll T_{c}$, increasing the effective spin relaxation leads to the formation of the peak in the temperature dependence of the dissipative part of the spin susceptibility. This peak is strongly suppressed by increasing the Dynes parameter, which models the smearing of the gap edge singularities in the superconductors due to the inhomogeneities or the inelastic phonon scattering.

Using this result and the model of interfacial exchange interaction, we examined the spin pumping from the ferromagnet with magnetization precession into the adjacent superconducting film. In the low-frequency regime, corresponding to recent experiments [11-19], we have analyzed the temperature dependence of the additional Gilbert damping parameter induced by the spin pumping. For realistic values of the Dynes parameter in materials such as $\mathrm{NbN}$ this temperature dependence is almost monotonic. This result indicates that there should exist some other mechanism for producing large peaks observed recently in S/FI structures [16]. The regime of large Dynes parameters can be also considered to model the spectral smearing which occurs due to the spatial inhomogeneity of the order parameter in systems with metallic ferromagnets. The monotonic suppression of the Gilbert damping parameter in this case corresponds to the experimentally observed behavior of FMR in Py/Nb systems [11]. Similar behavior is also reproduced by the scattering theory formalism [44].

For larger frequencies comparable with the superconducting gap and enhanced interfacial couplings, we get significant shifts of the FMR line. These shifts act towards increasing the resonant field $H_{0}$ at a given frequency. This behavior is opposite to the one found in recent experiments at low frequencies [14,17-19].

\section{ACKNOWLEDGMENTS}

This work was supported by the Academy of Finland (Project No. 297439) and the Russian Science Foundation, Grant No. 20-12-00053.

\section{APPENDIX A: ABSENCE OF SPIN RESPONSE WITHOUT SPIN RELAXATION}

In the absence of spin-orbit scattering, $\tau_{s o}^{-1}=0$ and $q=0$, the susceptibility can be written as follows:

$$
\chi_{h}(\Omega, q=0)=\pi T \sum_{\omega} \frac{\Delta^{2}+s_{1} s_{2}-\omega_{1} \omega_{2}}{s_{1} s_{2}\left(s_{1}+s_{2}\right)} .
$$

We can use the relations $s_{1}^{2}-s_{2}^{2}=\omega_{1}^{2}-\omega_{2}^{2}$ and $2\left(\omega_{1} \omega_{2}-\right.$ $\left.\Delta^{2}-s_{1} s_{2}\right)=\left(\omega_{1}+\omega_{2}\right)^{2}-\left(s_{1}+s_{2}\right)^{2}$, so that

$$
\begin{aligned}
& \sum_{\omega} \frac{\left(\omega_{1}+\omega_{2}\right)^{2}-\left(s_{1}+s_{2}\right)^{2}}{s_{1} s_{2}\left(s_{1}+s_{2}\right)} \\
& =\sum_{\omega}\left[\frac{\left(\omega_{1}+\omega_{2}\right)^{2}}{s_{1} s_{2}\left(s_{1}+s_{2}\right)}-\frac{s_{1}+s_{2}}{s_{1} s_{2}}\right] \\
& =\sum_{\omega}\left[\frac{\left(\omega_{1}+\omega_{2}\right)}{\left(\omega_{1}-\omega_{2}\right)}\left(s_{2}^{-1}-s_{1}^{-1}\right)-s_{1}^{-1}-s_{2}^{-1}\right] \\
& =-\frac{1}{\Omega} \sum_{\omega}\left[\left(\omega_{2}-\omega_{1}\right)\left(s_{1}^{-1}+s_{2}^{-1}\right)-\left(\omega_{1}+\omega_{2}\right)\left(s_{2}^{-1}-s_{1}^{-1}\right)\right] \\
& =\frac{2}{\Omega} \sum_{\omega}\left[\omega_{1} s_{1}^{-1}-\omega_{2} s_{2}^{-1}\right]=\frac{2}{\Omega} \sum_{\omega}\left[\operatorname{sgn}\left(\omega_{1}\right)-\operatorname{sgn}\left(\omega_{2}\right)\right] .
\end{aligned}
$$

Thus, after analytical continuation we can write

$$
\chi_{h}(\Omega)+1=\int_{-\infty}^{\infty} \frac{d \varepsilon}{2 \Omega}\left[n_{0}(\varepsilon+\Omega)-n_{0}(\varepsilon)\right]=1,
$$

so that $\chi_{h}(\Omega)=0$ at $\Omega \neq 0$. 


\section{APPENDIX B: NORMAL METAL LIMIT}

In the normal metal limit $\Delta=0$ and $\xi_{1,2}=\left|\omega_{1,2}\right|$. Then

$$
\chi_{h}+1=\pi T \sum_{\omega} \frac{1-\operatorname{sgn}\left(\omega_{1}\right) \operatorname{sgn}\left(\omega_{2}\right)}{\left(\left|\omega_{1}\right|+\left|\omega_{2}\right|+4 / 3 \tau_{s o}\right)} .
$$

Analytical continuation is implemented as follows:

$$
\begin{aligned}
\chi_{h} & +1 \\
& =\int_{-\infty}^{\infty} \frac{d \varepsilon}{4 i} \frac{\left[n_{0}(\varepsilon-1)-n_{0}(\varepsilon)\right]\left[1-\operatorname{sgn}\left(\omega_{1}\right)^{R} \operatorname{sgn}\left(\omega_{2}\right)^{A}\right]}{\left(\left|\omega_{1}\right|^{R}+\left|\omega_{2}\right|^{A}+4 / 3 \tau_{s o}\right)},
\end{aligned}
$$

where we have used $\left|\omega_{1}\right|^{R} \rightarrow s\left(-i \varepsilon_{1}^{R}\right)=i(\varepsilon-\Omega)+\Gamma$ and $\left|\omega_{2}\right|^{A} \rightarrow s\left(-i \varepsilon_{2}^{A}\right)=-i \varepsilon+\Gamma$, so that $\left|\omega_{1}\right|^{R}+\left|\omega_{2}\right|^{A} \rightarrow$ $-i(\varepsilon-\Omega)+i \varepsilon+2 \Gamma=i \Omega+2 \Gamma$.

Then we obtain

$$
\begin{aligned}
\chi_{h}+1 & =\int_{-\infty}^{\infty} \frac{d \varepsilon}{2 i} \frac{\left[n_{0}(\varepsilon)-n_{0}(\varepsilon+\Omega)\right]}{\left(i \Omega+2 \Gamma+4 / 3 \tau_{s o}\right)} \\
& =\frac{\Omega}{\Omega-2 i\left(2 / 3 \tau_{s o}+\Gamma\right)} .
\end{aligned}
$$

From this we obtain Eq. (22).

\section{APPENDIX C: DERIVATION OF THE STRONG SPIN RELAXATION LIMIT (26)}

Substituting Eq. (21), obtained assuming strong spin relaxation, into the general analytical continuation rule (14), we obtain

$$
\begin{aligned}
\frac{8}{3 i \tau_{s o}} \chi_{h}= & \Delta^{2} \int d \varepsilon\left[\frac{F_{1}(\varepsilon-\Omega)}{\xi^{A}(\varepsilon)}+\frac{F_{1}(\varepsilon+\Omega)}{\xi^{R}(\varepsilon)}\right] \\
& +\int d \varepsilon\left[\frac{F_{2}(\varepsilon-\Omega) \varepsilon}{\xi^{A}(\varepsilon)}+\frac{F_{2}(\varepsilon+\Omega) \varepsilon}{\xi^{R}(\varepsilon)}\right] \\
& +\int d \varepsilon\left[\frac{F_{2}(\varepsilon)(\varepsilon+\Omega)}{\xi^{A}(\varepsilon+\Omega)}-\frac{F_{2}(\varepsilon-\Omega) \varepsilon}{\xi^{A}(\varepsilon)}\right],
\end{aligned}
$$

where $\xi^{R, A}(\varepsilon)=\sqrt{\left(\varepsilon^{R, A}\right)^{2}-\Delta^{2}}, \quad F_{1}=n_{0}(\varepsilon) N(\varepsilon) / \varepsilon, \quad F_{2}=$ $n_{0}(\varepsilon) N(\varepsilon)$, and $N=\operatorname{Re}\left(\varepsilon / \xi^{R}\right)$ is the DOS. The contribution of the last term can be calculated to equal $-i \Omega$ using the asymptotic $F_{2}(\varepsilon \pm \infty)= \pm 1$ and $\varepsilon / \xi^{A}(\varepsilon) \rightarrow-1$ at large energies. The first two terms can be calculated using expansions $F(\varepsilon \pm \Omega)=F(\varepsilon) \pm \Omega \partial_{\varepsilon} F$, which yields

$$
\frac{2}{3 \tau_{s o}} \frac{\operatorname{Im} \chi_{h}}{\Omega}=\int_{-\infty}^{\infty} d \varepsilon \frac{N}{\varepsilon}\left(\Delta^{2} \partial_{\varepsilon} F_{1}+\varepsilon \partial_{\varepsilon} F_{2}\right)-1 .
$$

Integrating by parts this equation can be rewritten as Eq. (26) in the main text.

\section{APPENDIX D: CALCULATION OF LOCAL SPIN SUSCEPTIBILITY IN A FILM WITH FINITE THICKNESS}

To take into account finite metallic film thickness we incorporate the interfacial exchange field as the boundary conditions to the nonstationary Usadel equations:

$$
D \check{g} \circ \partial_{z} \check{g}(z=0)=i J_{s d}\left[\hat{\tau}_{3} \hat{\boldsymbol{\sigma}} \boldsymbol{m}, \hat{g}\right]_{t} .
$$

Mathematically, it is more convenient to consider the equivalent problem incorporating the interfacial exchange field as the point source to the Usadel equation:

$$
\begin{aligned}
- & i\left\{\hat{\tau}_{3} \partial_{t}, \check{g}\right\}_{t}+D \partial_{z}\left(\check{g} \circ \partial_{z} \check{g}\right) \\
& =i\left[\hat{\tau}_{3} \hat{\tau}_{2} \Delta, \check{g}\right]+\left[\check{\Sigma}_{s o} \circ, \check{g}\right]_{t}+i J_{s d} \delta(z)[\hat{m}, \check{g}]_{t} .
\end{aligned}
$$

This equation is considered in the interval $|z|<d_{M}$. If the interfaces with vacuum are at $z= \pm d_{M}$, the current vanishes,

$$
\check{g} \circ \partial_{z} \check{g}\left(z= \pm d_{M}\right)=0 .
$$

If the interfaces with very strong spin sink are at $z=d_{M}$, the correction to GF vanishes,

$$
\check{g}_{h}\left(z= \pm d_{M}\right)=0 .
$$

We assume that magnetization depends on time as $\boldsymbol{m}(t)=$ $\boldsymbol{m}_{\Omega} e^{-i \Omega t}$ and search for the corrections to the GF in the form

$$
\hat{g}\left(t, t^{\prime}\right)=T \sum_{\omega}\left[\hat{g}_{0}(1) e^{-i \omega_{1}\left(t_{1}-t_{2}\right)}+\hat{g}_{h}(12) e^{-i\left(\omega_{1} t_{1}-\omega_{2} t_{2}\right)}\right],
$$

where $\omega_{2}=\omega_{1}-\Omega$ and $\hat{g}_{h}$ represents the correction to the first order of the oscillating field $\boldsymbol{m}_{\Omega}$. To satisfy boundary conditions we search for the solution in the form

$$
\hat{g}_{h}(12)=\sum_{n=0}^{\infty} g_{q_{n}}(12) \cos \left(q_{n} z\right),
$$

with $q_{n}=n \pi / d_{M}$ in the case of the vacuum interface (D3) and $q_{n}=(n+1 / 2) \pi / d_{M}$ in the case of the strong spin sink interface (D4). Using the expansion $\delta(z)=\left(2 d_{M}\right)^{-1} \sum_{n} \cos \left(q_{n} z\right)$, we have the equation for the correction:

$$
\begin{aligned}
\left(\tilde{s}_{1}\right. & \left.+D q^{2}\right) \hat{g}_{0}(1) \hat{g}_{q}(12)-\tilde{s}_{2} \hat{g}_{q}(12) \hat{g}_{0}(2) \\
& =i\left(\boldsymbol{h}_{\Omega} \hat{\boldsymbol{\sigma}}\right)\left[\hat{g}_{0}(1) \hat{\tau}_{3}-\hat{\tau}_{3} \hat{g}_{0}(2)\right],
\end{aligned}
$$

where $\boldsymbol{h}_{\Omega}=\left(G_{i}^{\uparrow \downarrow} / 2 \nu d_{M}\right) \boldsymbol{m}_{\Omega}$. Using the commutation relation $\hat{g}_{0}(1) \hat{g}_{k}(12)+\hat{g}_{k}(12) \hat{g}_{0}(2)=0$, we get the solution given by

$$
\hat{g}_{q}(12)=i\left(\boldsymbol{h}_{\Omega} \hat{\boldsymbol{\sigma}}\right) \frac{\hat{\tau}_{3}-\hat{g}_{0}(1) \hat{\tau}_{3} \hat{g}_{0}(2)}{s_{1}+s_{2}+4 / 3 \tau_{s o}+D q^{2}} .
$$

The spin polarization at the $\mathrm{M} / \mathrm{F}$ interface can be written in terms of the susceptibility,

$$
\boldsymbol{S}(z=0)=v h_{\mathrm{eff}} \chi_{m}(\Omega) \boldsymbol{m}_{\Omega} .
$$

Substituting the solution (D8) into the expression for the spin polarization,

$$
\boldsymbol{S}(t, z)=-\left.i \frac{\pi v}{4} \operatorname{Tr}\left[\hat{\boldsymbol{\sigma}} \hat{\tau}_{3} \hat{g}\right]\right|_{t_{1,2}=t},
$$

we get the imaginary frequency local susceptibility of the finite-thickness film (39).
[1] J. Linder and J. W. A. Robinson, Nat. Phys. 11, 307 (2015).

[2] F. S. Bergeret, M. Silaev, P. Virtanen, and T. T. Heikkilä, Rev. Mod. Phys. 90, 041001 (2018).
[3] W. Han, S. Maekawa, and X.-C. Xie, Nat. Mater. 19, 139 (2020).

[4] C. Quay and M. Aprili, Philos. Trans. R. Soc. A 376, 20150342 (2018). 
[5] K. Ohnishi, S. Komori, G. Yang, K.-R. Jeon, L. Olde Olthof, X. Montiel, M. Blamire, and J. Robinson, Appl. Phys. Lett. 116, 130501 (2020).

[6] D. Beckmann, J. Phys.: Condens. Matter 28, 163001 (2016).

[7] M. Eschrig, Rep. Prog. Phys. 78, 104501 (2015).

[8] A. Brataas, Y. Tserkovnyak, G. E. W. Bauer, and B. I. Halperin, Phys. Rev. B 66, 060404(R) (2002).

[9] Y. Tserkovnyak, A. Brataas, and G. E. W. Bauer, Phys. Rev. Lett. 88, 117601 (2002).

[10] Y. Tserkovnyak, A. Brataas, G. E. W. Bauer, and B. I. Halperin, Rev. Mod. Phys. 77, 1375 (2005).

[11] C. Bell, S. Milikisyants, M. Huber, and J. Aarts, Phys. Rev. Lett. 100, 047002 (2008).

[12] K.-R. Jeon, C. Ciccarelli, H. Kurebayashi, L. F. Cohen, S. Komori, J. W. A. Robinson, and M. G. Blamire, Phys. Rev. B 99, 144503 (2019).

[13] K.-R. Jeon, C. Ciccarelli, H. Kurebayashi, L. F. Cohen, X. Montiel, M. Eschrig, S. Komori, J. W. A. Robinson, and M. G. Blamire, Phys. Rev. B 99, 024507 (2019).

[14] K.-R. Jeon, C. Ciccarelli, H. Kurebayashi, L. F. Cohen, X. Montiel, M. Eschrig, T. Wagner, S. Komori, A. Srivastava, J. W. A. Robinson, and M. G. Blamire, Phys. Rev. Appl. 11, 014061 (2019).

[15] K.-R. Jeon, C. Ciccarelli, A. J. Ferguson, H. Kurebayashi, L. F. Cohen, X. Montiel, M. Eschrig, J. W. A. Robinson, and M. G. Blamire, Nat. Mater. 17, 499 (2018).

[16] Y. Yao, Q. Song, Y. Takamura, J. P. Cascales, W. Yuan, Y. Ma, Y. Yun, X. C. Xie, J. S. Moodera, and W. Han, Phys. Rev. B 97, 224414 (2018).

[17] L.-L. Li, Y.-L. Zhao, X.-X. Zhang, and Y. Sun, Chin. Phys. Lett. 35, 077401 (2018).

[18] Y. Zhao, Y. Yuan, K. Fan, and Y. Zhou, Appl. Phys. Express 13, 033002 (2020).

[19] I. Golovchanskiy, N. Abramov, V. Stolyarov, V. Chichkov, M. Silayev, I. Shchetinin, A. Golubov, V. Ryazanov, A. Ustinov, and M. Y. Kupriyanov, Phys. Rev. Appl. 14, 024086 (2020).

[20] Y. Ohnuma, H. Adachi, E. Saitoh, and S. Maekawa, Phys. Rev. B 89, 174417 (2014).

[21] L. Hebel and C. P. Slichter, Phys. Rev. 113, 1504 (1959).

[22] C. Rettori, D. Davidov, P. Chaikin, and R. Orbach, Phys. Rev. Lett. 30, 437 (1973).
[23] L. Tagirov and K. Trutnev, J. Phys. F 17, 695 (1987).

[24] D. Inosov, J. Park, P. Bourges, D. Sun, Y. Sidis, A. Schneidewind, K. Hradil, D. Haug, C. Lin, B. Keimer et al., Nat. Phys. 6, 178 (2010).

[25] T. A. Maier and D. J. Scalapino, Phys. Rev. B 78, 020514(R) (2008).

[26] M. M. Korshunov and I. Eremin, Phys. Rev. B 78, 140509(R) (2008).

[27] A. V. Chubukov, D. V. Efremov, and I. Eremin, Phys. Rev. B 78, 134512 (2008).

[28] P. Hirschfeld, M. Korshunov, and I. Mazin, Rep. Prog. Phys. 74, 124508 (2011).

[29] M. Inoue, M. Ichioka, and H. Adachi, Phys. Rev. B 96, 024414 (2017).

[30] T. Taira, M. Ichioka, S. Takei, and H. Adachi, Phys. Rev. B 98, 214437 (2018).

[31] A. Abrikosov and L. Gor'kov, Sov. Phys. JETP 15, 752 (1962).

[32] R. C. Dynes, J. P. Garno, G. B. Hertel, and T. P. Orlando, Phys. Rev. Lett. 53, 2437 (1984).

[33] F. S. Bergeret and I. V. Tokatly, Phys. Rev. B 94, 180502(R) (2016).

[34] A. A. Abrikosov, L. P. Gorkov, and I. E. Dzyaloshinski, Methods of Quantum Field Theory in Statistical Physics (Courier Corporation, Chelmsford, MA, 2012).

[35] G. Eilenberger, Z. Phys. 214, 195 (1968).

[36] F. S. Bergeret, A. L. Yeyati, and A. Martín-Rodero, Phys. Rev. B 72, 064524 (2005).

[37] F. S. Bergeret, A. F. Volkov, and K. B. Efetov, Rev. Mod. Phys. 77, 1321 (2005).

[38] N. Kopnin, Theory of Nonequilibrium Superconductivity (Oxford University Press, Oxford, 2001).

[39] M. Silaev, Phys. Rev. B 99, 224511 (2019).

[40] M. Tinkham, Introduction to Superconductivity (Courier Corporation, Chelmsford, MA, 1996).

[41] Y. Masuda and A. Redfield, Phys. Rev. 125, 159 (1962).

[42] T. Kato, Y. Ohnuma, M. Matsuo, J. Rech, T. Jonckheere, and T. Martin, Phys. Rev. B 99, 144411 (2019).

[43] D. Davidov, C. Rettori, and H. Kim, Phys. Rev. B 9, 147 (1974).

[44] J. P. Morten, A. Brataas, G. E. Bauer, W. Belzig, and Y. Tserkovnyak, Europhys. Lett. 84, 57008 (2008). 